\title{
Abdominal visceral findings in patients with Marfan syndrome
}

Kira Chow, $M D^{1}$, Reed E. Pyeritz, $M D, P h D^{2,3}$, and Harold I. Litt, $M D, P h D^{1}$

\begin{abstract}
Purpose: Marfan syndrome is an autosomal dominant disorder historically defined by well-characterized features in the cardiovascular, ocular, and skeletal systems. To date, there have been no reports concerning abdominal visceral findings in this disorder. The purpose of this study was to determine the prevalence of abdominal visceral findings in patients with Marfan syndrome. Methods: Computed tomography or magnetic resonance studies of 69 patients with Marfan syndrome and an age- and sex-matched cohort of control subjects were reviewed. The presence of abdominal visceral findings was noted. Chi-square and Student $t$ tests were used to determine significance of differences between the patient and control groups. This retrospective study was approved by the local institutional review board and determined to be exempt from Health Insurance Portability and Accountability Act reporting requirements. Results: Renal cysts were present in 41 Marfan patients (59.4\%) versus 21 control subjects (30.4\%), $P=0.001$. The average number of renal cysts was greater in Marfan patients than controls $(2.4$ vs. 0.9, $P=0.005$ ). Hepatic cysts were present in 24 Marfan patients (34.8\%) versus 12 control patients (17.3\%), $P=0.02$. The average number of hepatic cysts was also greater in Marfan patients than controls $(0.9 \mathrm{vs} .0 .3$, $P=0.027)$. Cholelithiasis was present in 12 Marfan patients (18.1\%) versus one control patient $(1.5 \%), P<$ 0.001. Conclusions: Marfan syndrome patients have liver and renal cysts more often, in increased number, and at an earlier age than controls, in addition to an increased prevalence of cholelithiasis. Further study will be needed to relate these findings to recent developments concerning the underlying molecular genetics of this disorder.
\end{abstract}

Genet Med 2007:9(4):208-212.

Key Words: Marfan syndrome, renal cysts, hepatic cysts, cholelithiasis

Marfan syndrome is an autosomal dominant disorder historically defined by well-characterized features in the cardiovascular, ocular, and skeletal systems. ${ }^{1}$ In the recent past, additional organs and tissues have been found to be affected, such as the dura, lung, and skin, and diagnostic criteria have been expanded. ${ }^{2}$ All the features are, to a greater or lesser extent, age dependent, so older patients tend to have a more easily discernible phenotype. The natural history of the condition includes progressive dilation of the aortic root, which predisposes to dissection and aortic regurgitation. If untreated, the cardiovascular problems shorten life by one third to one half. ${ }^{3}$ Advances in medical and surgical management in the past 30 years have resulted in considerable improvement in life expectancy. ${ }^{4}$ It has become standard practice in our institution to image the chest, abdomen, and pelvis of adults with Marfan syndrome to

From the Departments of ${ }^{1}$ Radiology, ${ }^{2}$ Medicine, and ${ }^{3}$ Genetics, University of Pennsylvania School of Medicine, Philadelphia, PA.

Reed E. Pyeritz, MD, PhD, Division of Medical Genetics, Maloney 538, Hospital of the University of Pennsylvania, 3400 Spruce Street., Philadelphia, PA 19104-4283; E-mail: reed.pyeritz@uphs.upenn.edu

The authors declare no conflict of interest.

Submitted for publication September 6, 2006.

Accepted for publication January 31, 2007.

DOI: 10.1097/GIM.0b013e3180423cb3 detect, and if necessary follow, aortic and other vascular disease. The combination of longer life spans and more frequent imaging in Marfan syndrome patients has led to the recognition of additional features of the syndrome. ${ }^{5}$ An example is lumbosacral dural ectasia, the high prevalence of which became evident when patients were followed by computed tomography (CT) and magnetic resonance (MR) imaging of their entire aorta. In the course of reviewing studies of the chest, abdomen, and pelvis obtained for evaluation of the aorta, we incidentally noted that many patients had cysts in the liver and kidneys as well as other abdominal visceral findings. To our knowledge, there are no reports in the literature of increased incidence of renal, hepatic, or biliary findings in Marfan syndrome patients. The purpose of this study was to determine the prevalence of abdominal visceral findings in patients with Marfan syndrome.

\section{METHODS}

Imaging records of 89 patients meeting diagnostic criteria for Marfan syndrome ${ }^{2}$ followed in the medical genetics clinic of an academic tertiary care referral center were reviewed. A search of the radiology patient information system for CT or MR angiography examinations of the chest and/or abdomen obtained between January 1998 and March 2004 was per- 
formed. This time period was chosen because studies performed before January 1998 are not archived on our departmental picture archiving and communication system (PACS). This retrospective study was approved by the local institutional review board and determined to be exempt from Health Insurance Portability and Accountability Act reporting requirements.

Sixty-nine patients ( 28 female, 41 male; mean age, 44 years; range, 17-75 years) had undergone CT or MR angiography of the chest and/or abdomen in the given time period. In patients who had multiple studies, the most recent study was evaluated. Twenty-one CT and $48 \mathrm{MR}$ examinations were available for the 69 Marfan patients; 41 patients had dedicated abdominal imaging, and chest scans with imaging of the upper abdomen were reviewed in 28 .

A control population ( 28 females, 41 males; mean age, 44 years; range, $21-68$ years) was generated by searching the radiology information system for patients who were being evaluated as living related organ donors, using CT and MR for prospective renal donors, and MR only for prospective hepatic donors. All control subjects were purportedly healthy, and none had had imaging of the kidneys or liver before screening. Starting from June 2004, in reverse chronologic order to November 1999, all donor patients were matched by age within 1 year and gender with the study population. Any patients with known or suspected renal or hepatic disease were excluded. The mean age of 28 female Marfan syndrome patients was 43.6 years, and the mean age of 28 female control patients was 43.5 years. The mean age of 41 male Marfan syndrome patients was 44.6 years, and the mean age of 41 male control patients was 44.9 years. Dedicated abdominal CT $(n=16)$ or MR $(n=53)$ angiography studies were reviewed in the 69 control patients.

CT scans of the Marfan patients were performed on singleslice (4/21), four-slice (1/21), or 16-slice (16/21) CT scanners using dedicated angiographic technique including precontrast and thin-section angiographic phase imaging, with delayed parenchymal phase imaging in some patients. All CT scans of the donor control group were performed on a 16-slice CT scanner (Sensation 16, Siemens Medical Solutions, Malvern, PA) using a renal donor protocol, including precontrast-enhanced, thinsection, arterial phase and enhanced parenchymal phase imaging of the abdomen and pelvis. MR scans in patients and controls were obtained on 1.5-T magnets (LX series, General Electric, Milwaukee, WI or Siemens Symphony or Sonata) using local coils. The Marfan imaging protocol included axial gradient echo, T1-weighted in-phase and axial and coronal T2-weighted, single-shot, fast spin echo imaging of the chest. Abdominal imaging in the Marfan patients and renal and hepatic donor controls included axial gradient echo, T1weighted in- and out-of-phase images; coronal T2-weighted, single-shot, fast spin echo; and axial T2-weighted, fast-spin echo images with and without fat saturation. T1-weighted three-dimensional gradient echo images were obtained in an oblique sagittal plane of the chest and in a coronal plane of the abdomen before and after intravenous administration of 0.2 $\mathrm{mm} / \mathrm{kg}$ gadolinium diethylenetriaminepentaacetic acid. De- layed postcontrast axial T1-weighted, gradient echo images were also obtained, without fat saturation in the chest and with fat saturation in the abdomen.

CT and MR images were evaluated on a PACS workstation without multiplanar reformatting by one attending radiologist with 7 years experience reading CT and MR studies who was initially blinded to the reported findings. All original reports of the studies were then reviewed and images were evaluated again to confirm any findings in the study report that were not noted at initial blinded review. For CT studies, all imaging series were evaluated. For MR studies, all T2-weighted images were evaluated, as well as postcontrast axial T1-weighted images. The presence and number of abdominal visceral findings were noted, particularly renal cysts, hepatic cysts, and cholelithiasis. A cyst was defined as a round, hypoattenuating, nonenhancing lesion measuring $\geq 2 \mathrm{~mm}$ on CT and as a round T2-hyperintense, nonenhancing lesion measuring $\geq 2 \mathrm{~mm}$ on $\mathrm{MR}$, which was not clearly communicating with the collecting system.

Statistical analysis was performed using $\chi^{2}$ and $\chi^{2}$ Fisher exact tests (Stata Corporation, Version 5.0 College Station, TX) to determine the significance of differences in lesion prevalence. Paired $t$ tests (Microsoft Excel 2003, Redmond, WA) were used to determine the significance of differences in number of cysts.

\section{Table 1}

Age distribution and prevalence of renal cysts in Marfan patients versus control subjects

\begin{tabular}{lccc}
\hline Age, yr & Marfan patients & Control patients & $P$ \\
\hline$<40$ & $10 / 27(37 \%)$ & $4 / 25(16 \%)$ & 0.09 \\
$40-60$ & $24 / 33(73 \%)$ & $12 / 36(33 \%)$ & 0.001 \\
$>60$ & $7 / 9(78 \%)$ & $5 / 8(63 \%)$ & 0.5 \\
\hline
\end{tabular}

Table 2

Distribution of number of cysts in 69 Marfan patients versus 69 control subjects

\begin{tabular}{lccc}
\hline No. of renal cysts & Marfan patients & Control patients & $P$ \\
\hline 1 & $19(28 \%)$ & $11(15.9 \%)$ & 0.05 \\
2 & $1(1.4 \%)$ & $3(4.3 \%)$ & 0.25 \\
$>2$ & $21(30.4 \%)$ & $7(10.1 \%)$ & 0.002 \\
\hline
\end{tabular}

The number of cysts in the Marfan patients ranged from 0 to 27 . Note in particular the increased number of Marfan patients with more than two cysts.

Table 3

Age distribution and prevalence of hepatic cysts in Marfan patients versus control subjects

\begin{tabular}{lccc}
\hline Age, yr & Marfan patients & Control patients & $P$ \\
\hline$<40$ & $7 / 27(26 \%)$ & $4 / 25(16 \%)$ & 0.4 \\
$40-60$ & $15 / 33(45 \%)$ & $6 / 36(17 \%)$ & 0.009 \\
$>60$ & $2 / 9(22 \%)$ & $2 / 8(25 \%)$ & 0.9 \\
\hline
\end{tabular}



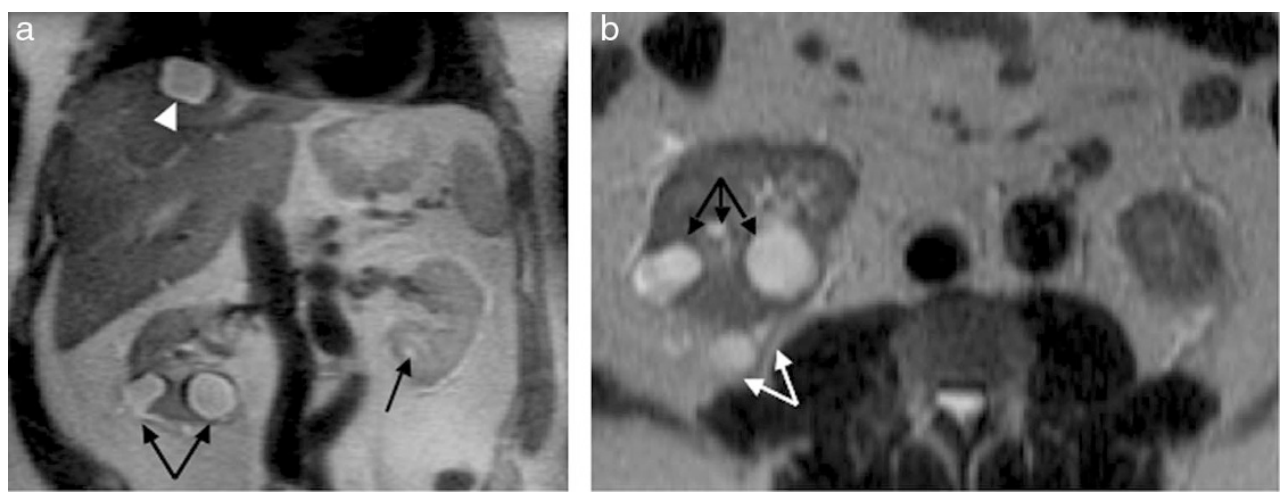

Fig. 1. A 55-year-old male Marfan syndrome patient with 11 renal cysts and 7 hepatic cysts. Coronal single-shot, fast spin echo, T2-weighted image (a) demonstrates hepatic cyst (arrowhead) and bilateral renal cysts (arrows). Axial single-shot, fast spin echo, T2-weighted image (b) demonstrates multiple right renal cysts (arrows).

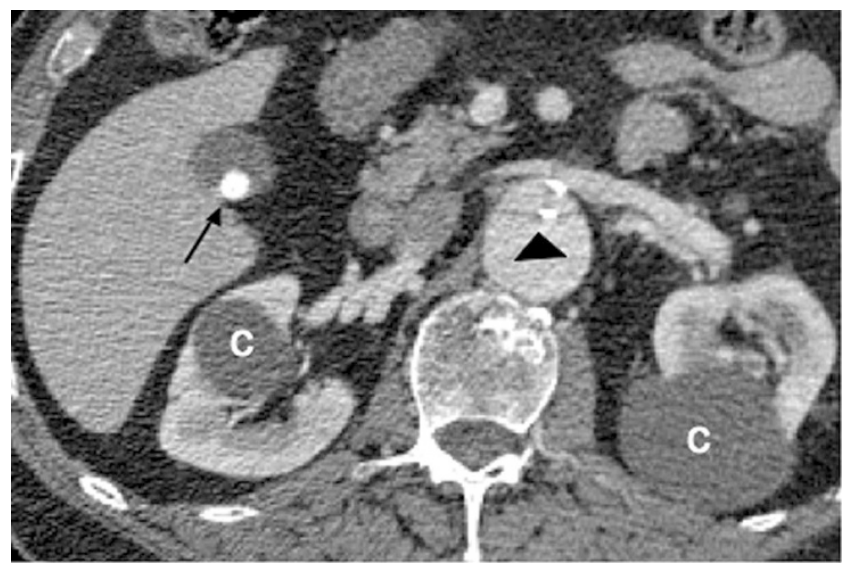

Fig. 2. A 70-year-old male Marfan syndrome patient with cholelithiasis (arrow) and bilateral renal cysts (C) demonstrated on angiographic phase of CT study. Dissection flap in the abdominal aorta is also visualized (arrowhead).

\section{RESULTS}

Renal cysts were present in 41 Marfan patients (59.4\%) versus 21 control subjects $(30.4 \%) P=0.001$. The prevalence of renal cysts was stratified by age (Table 1) and significantly greater in Marfan patients aged 40-60 years than controls $(P=0.001)$ and displayed a trend toward a greater prevalence in patients younger than 40 years $(P=0.09)$. The average number of renal cysts was greater in Marfan patients than controls (2.4 vs. $0.9, P=0.005)$. The numbers of cysts per subject are shown in Table 2. The number of cysts in Marfan patients ranged up to 27, with 10 patients having six or more.

Hepatic cysts were present in 24 Marfan patients (34.8\%) versus 12 control patients $(17.3 \%), P=0.02$. The prevalence of hepatic cysts was stratified by age (Table 3 ) and significantly greater in Marfan patients aged 40-60 years than controls $(P=0.009)$. The average number of hepatic cysts was also greater in Marfan patients than controls ( 0.9 vs. $0.3, P=0.027$ ).

Cholelithiasis was present in 12 Marfan patients (18.1\%) versus one control patient $(1.5 \%) P<0.001$. Patients with previous cholecystectomy in both groups (three Marfan patients, one control patient) were excluded from analysis.

Figures 1-4 show examples of abdominal visceral as well as typical vascular findings in Marfan patients.

\section{DISCUSSION}

The criteria for diagnosis of Marfan syndrome include anomalies of the skeletal, ocular, cardiovascular, and pulmonary systems as well as dural ectasia and striae atrophicae. Additional features found more often in people with Marfan syndrome, which have not yet been introduced as diagnostic criteria, include skeletal muscle hypoplasia, a paucity of peripheral adiposity, reduced bone density, and attention-deficit/hyperactivity disorder. To date, there have been no reports of abdominal visceral findings in Marfan patients. Two reports have described one family and one patient with coexistent autosomal dominant polycystic kidney disease (APKD) and Marfan syndrome, $, 6,7$ but these likely represent coincidence. In the family studied most extensively, the APKD phenotype was linked to PKD1 and was inherited from one branch of the family, while the Marfan phenotype was linked to FBN1 and derived from another branch. ${ }^{7}$

The results of our study suggest that patients with Marfan syndrome have an increased prevalence of renal and hepatic cysts and that they tend to develop these cysts at an earlier age than control subjects. The number of renal and hepatic cysts per patient is also significantly increased compared to the control population. In fact, there are several reasons that our study may somewhat underestimate the incidence of renal and hepatic cysts in Marfan patients relative to control patients. First, several of the CT studies in Marfan patients were performed on older single- or four-slice CT scanners, whereas all control subjects were imaged using a 16-slice scanner. The older scanners may be less sensitive for detecting small cysts. Also, more Marfan patients were studied by CT compared with MR than in the control population, and CT likely has a lower sensitivity compared with MR for detection of visceral cysts, given the greater contrast between organ parenchyma and fluid on T2weighted MR images. Additionally, all control subjects had dedicated abdominal imaging studies compared with only $70 \%$ (48/69) of Marfan patients; abdominal findings may have been less conspicuous on dedicated studies of the chest.

The prevalence of renal and hepatic cysts in the normal population in previously published studies is affected by multiple variables, including age of the patient population, imaging modality, and technical quality of the equipment used. The prev- 

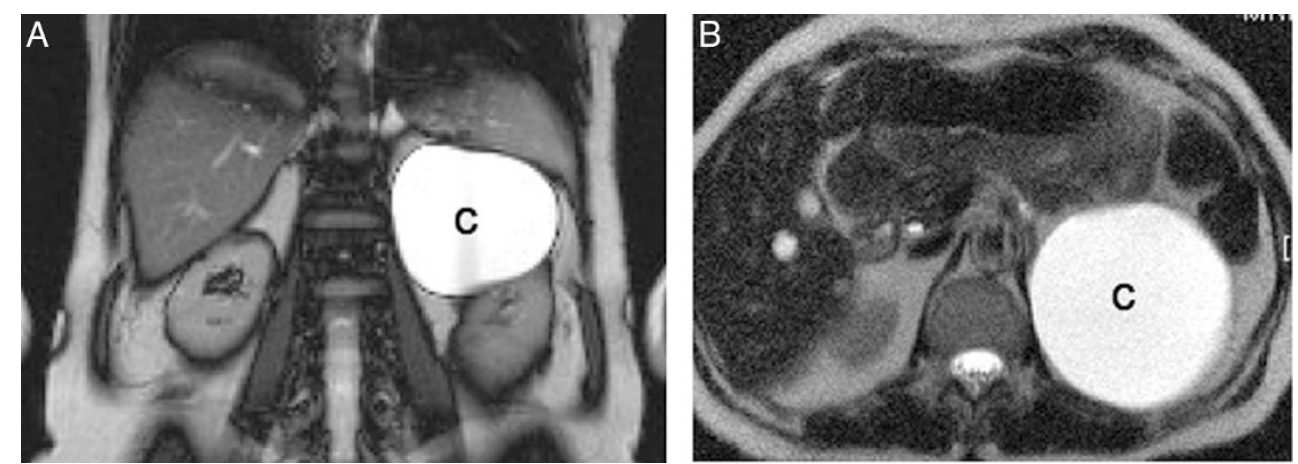

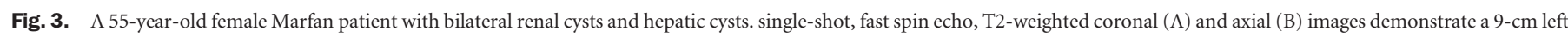
renal cyst (C).
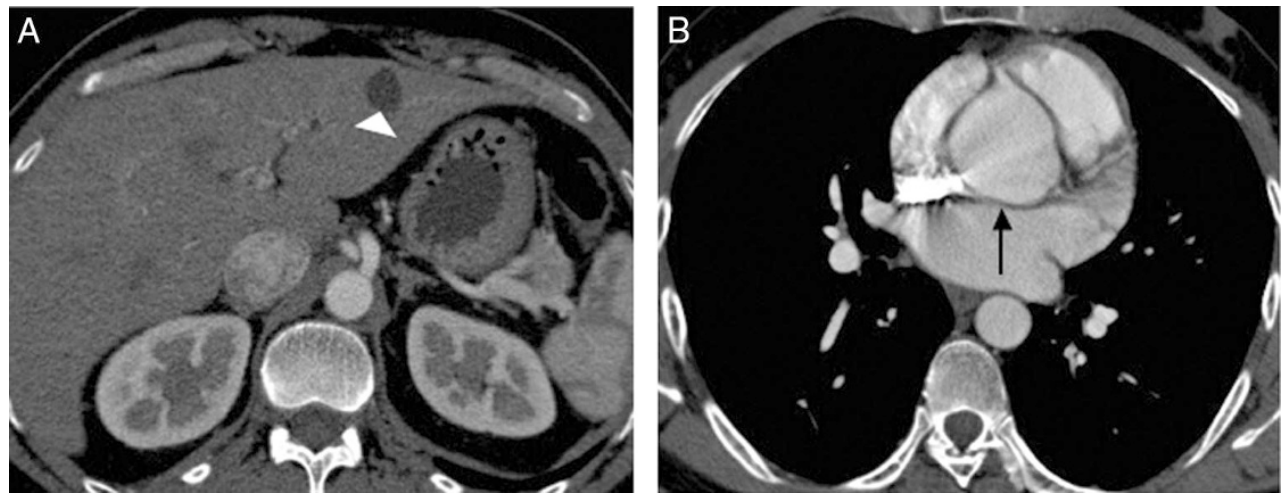

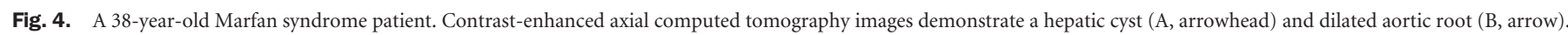

alence of hepatic cysts in the normal population in previously published studies ranges from $2 \%$ to $5 \%$ in ultrasound studies $^{8,9}$ and from $14 \%$ to $18 \%$ in CT studies, ${ }^{10,11}$ which is similar to our control population prevalence of $17 \%$. The prevalence of renal cysts in the normal population has a wider range. For example, in two recent studies, Terada et al. ${ }^{12}$ reported an $11.9 \%$ prevalence of renal cysts by ultrasonography in more than 14,000 patients with a mean age of 52.1 years, whereas Carrim and Murchison ${ }^{10}$ found renal cysts in $41 \%$ of 617 patients with a mean age of 62.8 years in females and 65.5 years in males. A recent study of renal cysts using MR imaging reported a prevalence of $62.5 \%$ in a population with average age of older than 50 and no subjects older than 59 years old. ${ }^{13}$ Our control population had a lower mean age than either that of these studies ( 44 years) because it was age matched to the Marfan syndrome population. Marfan syndrome patients tend to be younger because they come to medical attention earlier. Another difference between our control population and those in other prevalence studies was that our population was restricted to renal and hepatic donors who did not have comorbid conditions, which could potentially increase the prevalence of renal cysts, hepatic cysts, or cholelithiasis.

Previous studies have repeatedly found that the prevalence and number of renal ${ }^{10,12,14,15}$ and hepatic ${ }^{9-11}$ cysts increases with age. In both our Marfan patient population and our control population, the prevalence of renal and hepatic cysts increased with age, but when stratified by age, there was a signif- icant difference in prevalence of both renal and hepatic cysts between our two groups of patients aged $40-60$ years. We did not find a significant difference in the two groups in patients younger than 40 years, probably because of the small number of patients in this group $(N=27)$. The high prevalence of renal and hepatic cysts in control subjects older than 60 years and the small numbers of patients of this age in our study likely explain the lack of a significant difference in this age group.

We hypothesize that earlier formation of renal and hepatic cysts and increased number of cysts may be explained by a different pathogenesis of cyst formation in Marfan patients. In the general population, renal cysts are thought to be acquired lesions originating as diverticula on the distal tubules due to weakening of the basement membrane, more likely in older patients or those with previous urinary obstruction. $.^{10,14,16} \mathrm{He}-$ patic cysts are thought to occur as a result of congenitally aberrant bile ducts, which are more prone to biliary stasis or obstruction, eventually resulting in dilation and cyst formation.9,10 Marfan syndrome has long been characterized as a heritable disorder of connective tissue, and the discovery of its cause, mutations in the gene encoding the extracellular matrix protein fibrillin-1, seemingly supported that categorization. However, recent studies of pathogenesis of Marfan syndrome in mice engineered to harbor a fibrillin-1 mutation known to cause the human condition have dramatically shifted perceptions. The extracellular microfibrils, of which fibrillin is the major component, are important regulators of activity of the cytokine 
transforming growth factor $\beta$ (TGF $\beta$ ) family. Thus far, classic features of Marfan syndrome in the lung, mitral valve, and aortic wall have been documented to be due to increased activity of TGF $\beta 1$ during development and early growth. ${ }^{17-19}$ Whether visceral cyst formation in Marfan syndrome is influenced by TGF $\beta 1$ or reflects a structural failing of microfibrils remains to be investigated. Interestingly, one murine model of Marfan syndrome did not show renal involvement, but the fibrillin mutation, which resulted in a hypomorphic allele, was not of a type typically found in human patients. ${ }^{20}$ The kidneys and livers in murine models of Marfan syndrome generated by introducing missense mutations found in humans have not been examined.

Neither we nor others have explored the occurrence of renal and hepatic cysts in other heritable disorders of connective tissue. In most such conditions, CT or MR imaging of the abdomen is not routinely necessary, so a subject population would need to be investigated under a research protocol. We reviewed the CT and MR images of several patients with the vascular type of EhlersDanlos syndrome and did not find any cysts.

We found an increased prevalence of cholelithiasis in Marfan patients. This is probably underestimated by our study because the three Marfan syndrome patients and one control subject who had undergone cholecystectomy were excluded. These patients likely also had cholelithiasis, which would further increase the relative frequency in Marfan patients. In the normal population, reported prevalence of cholelithiasis varies widely, with one important variable being the study population's country of origin. The etiology of the increased prevalence of cholelithiasis in Marfan patients is not clear at present.

Potential limitations of this study include the size of the study population and choice of the potential renal and hepatic donor population as representative of normal subjects.

In conclusion, Marfan syndrome patients have liver and renal cysts more often, in increased number, and at an earlier age than controls. The etiology of this finding is uncertain, but in the kidney may be related to dilation of renal tubules. Many issues remain to be studied, including the time course of appearance and growth rate of cysts compared with established growth rates in the general population. Perhaps most interesting will be investigating the pathogenesis of the cysts and cholelithiasis. Future directions include histopathologic specimen analysis to determine gallstone composition and investigation of additional variables that may be associated with gallstone formation.

\section{References}

1. Pyeritz RE. Marfan syndrome and other disorders of fibrillin. In: Rimoin DL, Conner JM, Pyeritz RE, Korf B, editors. Principles and practice of medical genetics, 5 th ed. Edinburgh: Churchill Livingstone, 2007; 3579-3624, in press.

2. De Paepe A, Devereux RB, Dietz HC, Hennekam RC, et al. Revised diagnostic criteria for the Marfan syndrome. Am J Med Genet 1996;62:417-426.

3. Murdoch JL, Walker BA, Halpern BL, Kuzma JW, et al. Life expectancy and causes of death in the Marfan syndrome. N Engl J Med 1972;286:804-808.

4. Silverman DI, Burton KJ, Gray J, et al. Life expectancy in the Marfan syndrome. Am J Cardiol 1995;75:157-160.

5. Pyeritz RE, Fishman EK, Bernhardt BA, Siegelman SS. Dural ectasia is a common feature of the Marfan syndrome. Am J Hum Genet 1988;43:726-732.

6. Biermann CW, Rutishauser G. Polycystic kidneys associated with Marfan syndrome in an adult. Scand J Urol Nephrol 1994;28:295-296.

7. Hateboer N, Buchalter M, Davies SJ, Lazarou LP, et al. Co-occurrence of autosomal dominant polycystic kidney disease and Marfan syndrome in a kindred. Am J Kidney Dis 2000;35:753-760.

8. Huang JF, Chen SC, Lu SN, Lin ZY, et al. Prevalence and size of simple hepatic cysts in Taiwan: community- and hospital-based sonographic surveys. Gaoxiong Yi Xue Ke Xue Za Zhi 1995;11:564-567.

9. Gaines PA, Sampson MA. The prevalence and characterization of simple hepatic cysts by ultrasound examination. Br J Radiol 1989;62:335-337.

10. Carrim ZI, Murchison JT. The prevalence of simple renal and hepatic cysts detected by spiral computed tomography. Clin Radiol 2003;58:626-629.

11. Volk M, Strotzer M, Lenhart M, Techert J, et al. Frequency of benign hepatic lesions incidentally detected with contrast-enhanced thin-section portal venous phase spiral CT. Acta Radiol 2001;42:172-175.

12. Terada N, Ichioka K, Matsuta Y, Okubo K, et al. The natural history of simple renal cysts. J Urol 2002;167:21-23.

13. Nascimento AB, Mitchell DG, Zhang XM, Kamishima T, et al. Rapid MR imaging detection of renal cysts: age-based standards. Radiology 2001;221:628-632.

14. Laucks SP, McLachlan MS. Aging and simple cysts of the kidney. Br J Radiol 1981; 54:12-14.

15. Marumo K, Horiguchi Y, Nakagawa K, Oya M, et al. Incidence and growth pattern of simple cysts of the kidney in patients with asymptomatic microscopic hematuria. Int J Urol 2003;10:63-67.

16. Pederson JF, Emamian SA, Nielson MB. Simple renal cyst: relations to age and arterial blood pressure Br J Radiol 1993;66:581-584.

17. Neptune ER, Frischmeyer PA, Arking DE, Myers L, et al. Dysregulation of TGF-beta activation contributes to pathogenesis in Marfan syndrome. Nat Genet 2003;33:407411.

18. Ng CM, Cheng A, Myers LA, Martinez-Murillo F, et al. TGF-beta-dependent pathogenesis of mitral valve prolapse in a mouse model of Marfan syndrome. J Clin Invest 2004;114:1586-1592.

19. Habashi JP, Judge DP, Holm TM, Cohn RD, et al. Losartan, an AT1 antagonist, prevents aortic aneurysm in a mouse model of Marfan syndrome. Science 2006;312: $117-121$.

20. Hartner A, Eifert T, Haas CS, Tuysuz C, et al. Characterization of the renal phenotype in a mouse model of Marfan syndrome. Virchows Arch 2004;445:382-388. 